\title{
Optimization of Image Contrast for Size Distribution Measurements of Nanomaterials by Transmission Electron Microscopies Including TEM and T-SEM
}

\author{
Kazuhiro Kumagai, Shin-ya Terauchi and Akira Kurokawa \\ Surface and Nano-Analysis Section, Nanomaterial Characterization Division, National Metrology \\ Institute of Japan, National Institute of Advanced Industrial Science and Technology (AIST), Tsukuba \\ Japan
}

The regulations on nanomaterials, which started in several EU countries based on the definition of nanomaterial by European committee [1], have now become important issue in nano-related industry. Because, to check if a specimen meets the definition or not, we need to measure its size distribution to count the number of particles of $1-100 \mathrm{~nm}$ in diameter. Due to technical difficulties, there are increasing discussions to find the solution for the size distribution measurements [2]. In some possible methods, transmission electron microscopy (TEM) and scanning electron microscopy (SEM) are regarded as effective tools due to their capability to easily and quickly observe individual nano-particles [3].

To achieve reliable particle analysis with the electron microscopies (EMs), micrographs should show enough contrast to identify nano-particles. Since EM image depends on the primary electron (PE) energy [4], we should choose appropriate observing condition, in particular, low atomic number materials that generally give low contrast. There are, however, not so many practical discussions of image quality and observing condition for nano-particle counting. In this paper, we report the PE energy dependence of visibility of nano-particles over wide energy range by using TEM and SEM in transmitting mode (T-SEM).

A model specimen was made by dropping the mixture of suspensions of polystyrene spheres $(260 \mathrm{~nm}$ and $30 \mathrm{~nm}$ in diameter) onto a $\mathrm{Cu}$ grid with carbon support. We observed this specimen with three different PE energy conditions, at $300 \mathrm{keV}$ (TEM; JEM-3000F, JEOL), $120 \mathrm{keV}$ (TEM; JEM-1400 Plus, JEOL) and $20 \mathrm{keV}$ (T-SEM; JSM-7100F, JEOL).

For PE energy of $300 \mathrm{keV}$, the polystyrene spheres in the both size shown low contrast against background, though the image was taken under defocus condition [Fig. 1(a)]. The image at $120 \mathrm{keV}$, 260-nm spheres become darker than 30-nm spheres due to larger electron-solid interaction [Fig. 1(b)]. When it comes to $20 \mathrm{keV}$, even the $30-\mathrm{nm}$ particles are imaged with high contrast against background [Fig. 2(a)]. It should be pointed out that many minute particles smaller than $30 \mathrm{~nm}$ are visualized in 20 $\mathrm{keV}$ image, and are not artifacts come from carbon support [Fig. 2(b)]. Although these might be byproduct of polystyrene spheres, what important is that these are not observed at all in the other two images. These figures clearly demonstrate that PE energy condition easily changes the result of size distribution analysis with EMs.

To establish reliable size distribution measurements of nanomaterials by EMs, we have to take into account not only image resolution, but also image contrast formation over wide range of PE energies from TEM to SEM. To avoid counting loss of tiny particles, low voltage observation such as T-SEM is useful especially for low atomic number specimens. In the presentation, we would like to discuss the practical limitations in TEM and T-SEM to image nano-paticles. 


\section{References:}

[1] European Commission, Official Journal of the European Union L 275, 20.10.2011 (2011) p. 38. [2] A Lopez-Serrano et al, Analytical Methods 6 (2014) p. 38. [3] EFSA Scientific Committee, EFSA Journal 9 (2011) 2140.

[4] L Reimer and H Kohl, Transmission Electron Microscopy: Physics of Image Formation (Springer, 2008).

[5] The authors thank Dr. H. Kato in AIST for kind help in specimen preparation. This work was performed as one of the studies conducted by the consortium for measurement solutions for industrial use of nanomaterials (COMS-NANO) in Japan.
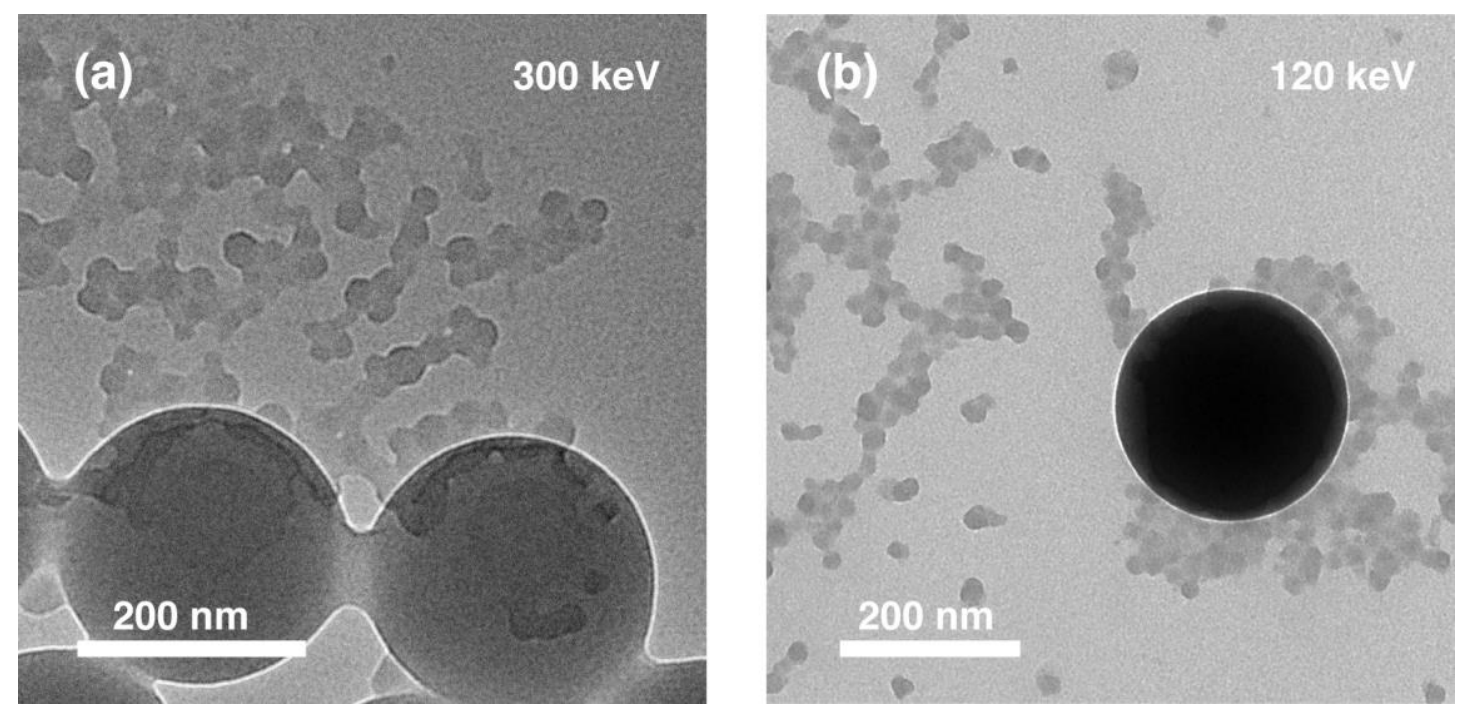

Figure 1. (a) A TEM bright field image of polystyrene spheres taken with PE energy of $300 \mathrm{keV}$. (b) The same as (a), but PE energy was $120 \mathrm{keV}$.
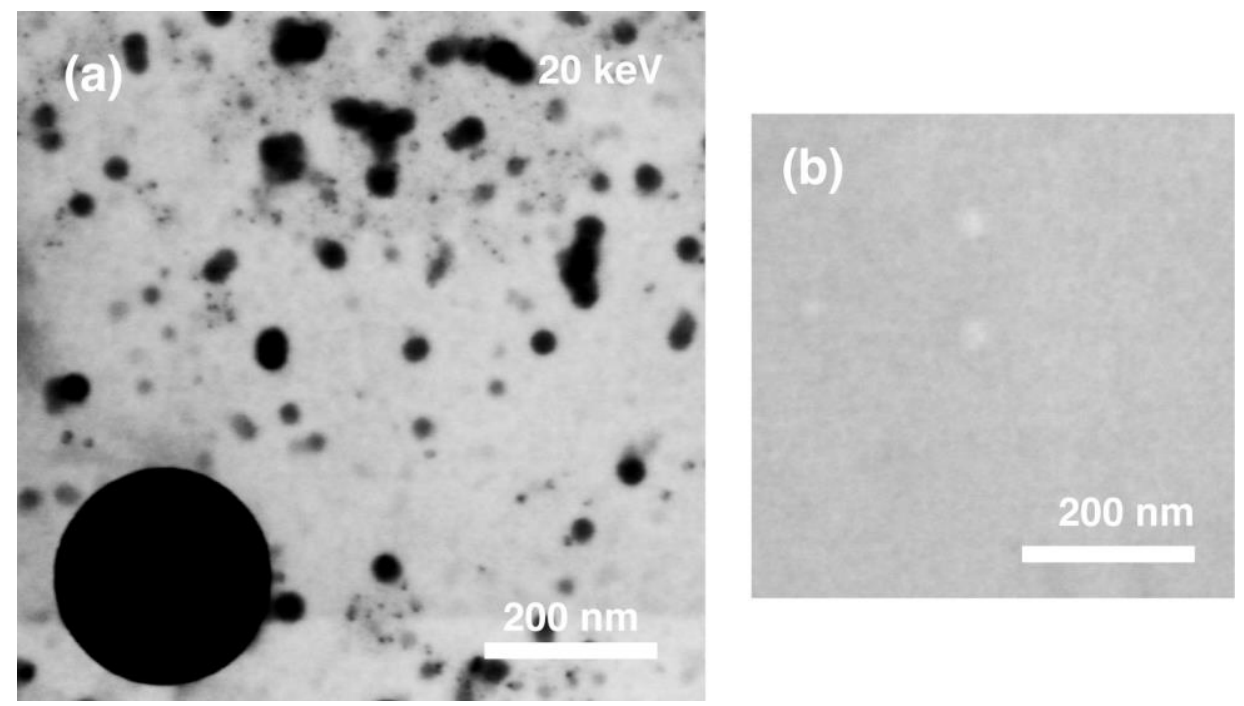

Figure 2. (a) A T-SEM bright field image of polystyrene spheres taken with T-SEM at PE energy of 20 $\mathrm{keV}$. Tiny particles smaller than $30 \mathrm{~nm}$ are observed. (b) A T-SEM bright field image of blank area in $\mathrm{Cu}$ grid. 